\title{
Review Article \\ MicroRNAs in Coronary Heart Disease: Ready to Enter the Clinical Arena?
}

\author{
Elena Cavarretta ${ }^{1}$ and Giacomo Frati ${ }^{1,2}$ \\ ${ }^{1}$ Department of Medical-Surgical Sciences and Biotechnologies, Sapienza University of Rome, \\ Corso della Repubblica 79, 04100 Latina, Italy \\ ${ }^{2}$ Department of AngioCardioNeurology, IRCCS NeuroMed, 86077 Pozzilli, Italy
}

Correspondence should be addressed to Elena Cavarretta; elena.cavarretta@gmail.com

Received 11 August 2016; Accepted 25 September 2016

Academic Editor: Ling-Qing Yuan

Copyright (C) 2016 E. Cavarretta and G. Frati. This is an open access article distributed under the Creative Commons Attribution License, which permits unrestricted use, distribution, and reproduction in any medium, provided the original work is properly cited.

\begin{abstract}
Coronary artery disease (CAD) and its complication remain the leading cause of mortality in industrialized countries despite great advances in terms of diagnosis, prognosis, and treatment options. MicroRNAs (miRNAs), small noncoding RNAs, act as posttranscriptional gene expression modulators and have been implicated as key regulators in several physiological and pathological processes linked to CAD. Circulating miRNAs have been evaluated as promising novel biomarkers of CAD, acute coronary syndromes, and acute myocardial infarction, with prognostic implications. Several challenges related to technical aspects, miRNAs normalization, drugs interaction, and quality reporting of statistical multivariable analysis of the miRNAs observational studies remain unresolved. MicroRNA-based therapies in cardiovascular diseases are not ready yet for human trials but definitely appealing. Through this review we will provide clinicians with a concise overview of the pros and cons of microRNAs.
\end{abstract}

\section{Introduction}

Approximately every 42 seconds, a US American will suffer a heart attack. Cardiovascular and cerebrovascular diseases represent the leading cause of death worldwide, even if death rates have fallen from 1968 to the present [1]. Moreover, the lifetime risk for coronary heart disease varies drastically as a function of risk factor profile. With an optimal risk factor profile, lifetime risk for CHD is $3.6 \%$ for men and $<1 \%$ for women; with $\geq 2$ major risk factors, it is $37.5 \%$ for men and $18.3 \%$ for women [2]. Therefore, a correct identification of those individuals by specific biomarkers related to diagnosis, screening, staging, monitoring, surveillance, prognosis, and treatment selection would be of pivotal importance. Genetics, intermediate phenotype, life-style, and other environmental triggers are directly involved in the pathogenesis of coronary artery disease (CAD). The estimated heritability of CAD ranges from 30 to $60 \%[3,4]$. Recently, several studies highlighted that the genetics of CAD largely derives from the cumulative effect of multiple common risk alleles, emphasizing the individual but cumulative small effect size rather than rare variants with large effects on CAD risk. Despite this finding, there has been less success in understanding the function of the novel loci; in fact the majority of these loci are in noncoding regions of the genome [5]. Even if most of our genome does not encode for proteins and it is extensively transcribed anyway, generating non(protein) coding RNAs. Short noncoding RNAs of approximately 2224 nucleotides, microRNAs, are widely recognized posttranscriptional gene regulator, while longer (>200 nucleotides) noncoding RNAs are now also recognized to play important roles in gene regulation and function [6]. MicroRNAs in cardiovascular disease are gaining momentum as possible novel biomarkers in the diagnosis and prognosis of coronary artery disease, acute coronary syndrome, and heart failure. Nowadays, diagnosis of acute coronary syndrome relies on symptoms, electrocardiogram abnormalities, and troponin quantification, with much interest in developing new rule-out and rule-in strategies or possible new promising biomarkers. In the literature, there is much ado about a possible clinical role of microRNAs in coronary heart disease. We aimed to review the pros and cons of microRNAs use in coronary heart disease applied to the clinical setting. 


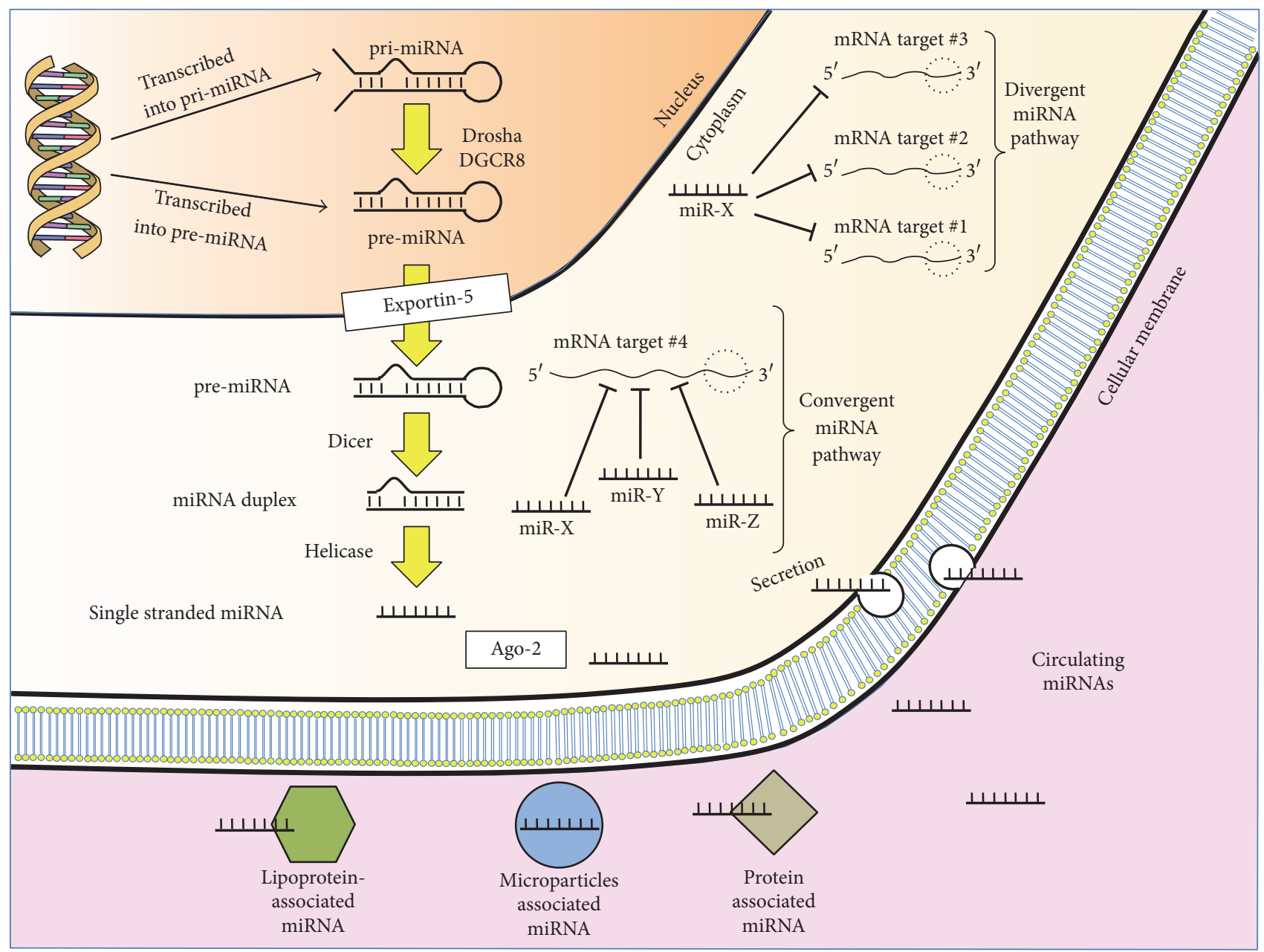

FIGURE 1: MicroRNAs biogenesis and function. In the nucleus, DNA is transcribed into pri-miRNA and then cleaved by Drosha to produce pre-miRNA in the canonical microRNA biogenesis pathway. Noncanonical biogenesis pathways exist. Pre-miRNA is then moved in the cytoplasm by Exportin- 5 where another RNase III, Dicer cleaves it into a microRNA duplex, to finally obtain a single stranded microRNA. The microRNA can exercise his action internally or in a cell-to-cell interaction, through convergent or divergent microRNA pathways. Circulating microRNAs are usually associated with lipoprotein, protein, exosome, and microvesicles. See text for further details. Ago-2: Argonaute protein 2; miRNA: microRNA; mRNA: messenger RNA.

\section{MicroRNAs}

2.1. MicroRNA Biology. In 1993, Lee et al. [7] discovered that the C. elegans gene lin-4 (a gene controlling the nematode larval development) did not encode for a protein but for small noncoding RNAs. A longer one (61 nucleotides) was then cleaved and folded in a stem-loop of 22 nucleotides. This lin4 derived RNAs had antisense complementarity to multiple sites in the $3^{\prime}$-UTR of the lin-14 gene, with a final result of reducing the amount of LIN-14 protein, without changing the amount of lin-14 messenger RNA (mRNA) [8]. This short lin4 RNA is the founding member of the microRNAs family. The second member of this family, let-7, had to wait until the year 2000 to be described by Reinhart et al. [9]. Since then the miRNAs family has markedly expanded and more than 2000 different miRNAs sequences have been described and catalogued in miRBase [10]. MicroRNAs function as gene regulators acting on mRNAs translation, with inhibition of protein synthesis. Basically, different miRNAs may target a given mRNA in different binding sites (convergent microRNA pathway) or a single miRNA may target multiple different mRNAs (divergent miRNA pathway) [11]. There are specific types of software to predict which mRNAs may be the target of a specific miRNA (TargetScan, http://www.targetscan.org/; miRanda, http://www.microRNA.org/; TarBase (http://www .microrna.gr/tarbase).

MicroRNAs biogenesis is resumed in Figure 1. Briefly, in the canonical miRNAs biogenesis pathway, primary miRNAs (pri-miRNAs) of hundreds or thousands of nucleotides are synthesized from DNA by the enzyme RNA polymerase II in the nucleus. Pri-miRNAs, folded in the hairpin structure, are then cleaved by the ribonuclease III Drosha with the cofactor DGCR8, to form the microprocessor complex, producing the preliminary miRNAs (pre-miRNAs) of 70-100 nucleotides. The pre-miRNAs are transported into the cytoplasm by Exportin-5 where another ribonuclease III, Dicer, and its 
cofactor TRBP, cleave them into the shorter, double stranded immature microRNAs. The miRNA-miRNA* duplex is then transferred to the Argonaute protein family (Ago) that undergoes conformational changes to allow binding of the miRNAs duplex. In the strand selection process, the passenger strand or $\mathrm{miR}^{*}$ is discarded while the leading strand or miR is incorporated into the RISC (RNA-Induced Silencing Complex). Into the RISC, the miRNA presents the seed sequence at an interface where it can interact with a region of the mRNA within its $3^{\prime}$-UTR [12]. Other noncanonical miRNAs biogenesis pathways have been described [13].

2.2. Circulating MicroRNA. MicroRNAs can act intracellularly or can be actively secreted by cells and contribute to intercellular or cell-tissue communication [14]. Circulating microRNAs are stable despite the high extracellular RNase activity, due to their packaging in apoptotic bodies, microvesicles, and exosomes or association with lipoprotein, protein as the Argonaute family and other RNA binding proteins. Microvesicles and exosomes are fundamentally different, the first being smaller and heterogeneous in size, ranging from $100 \mathrm{~nm}$ to $1 \mu \mathrm{m}$, derived from the plasma membrane and released by budding and fission of the membrane, while the latter being formed intracellularly via endocytic invagination and then released into a multivesicular body [15]. Since the discovery in 2008 of miRNAs in blood [16], circulating miRNAs have been found in blood, urine, breast milk [17], saliva [18], tears, and other body fluids [19]; their potential use as serum biomarkers has become more appealing. Biomarkers should be divided into two different categories, depending on their possibility to change over time: genetic markers, stable over time, and dynamic markers, which may change mainly over time. A biomarker should be noninvasively obtained and have a high degree of sensitivity and specificity, permitting early diagnosis of disease. Moreover, a biomarker should have time-related changes in the disease course, a long half-life within the sample, allowing rapid and cost-effective laboratory detection. Some of these essential characteristics are shared by circulating miRNAs: their small size, a simple chemical composition, their high stability in boiling water, their resistance to extreme $\mathrm{pH}$ changes, prolonged room temperature stays or repeated freeze-thawing $[19,20]$, less complexity in comparison with proteins, and a cost-effective quantification by real-time polymerase chain reaction (qRTPCR).

\section{MicroRNAs in Coronary Heart Disease}

3.1. MicroRNAs in Acute Coronary Syndrome. In 2010, five authors [21-25] independently reported a possible role for cardiomyocyte-enriched miRNAs in the diagnosis of acute myocardial infarction (AMI). Specifically, in these studies taken together, miR-1, miR-133a, miR-133b, miR-208, and miR-499 were found upregulated in plasma of AMI patients. Figure 2 resumes the potential miRNAs up- and downregulated in AMI with diagnostic and prognostic implications. More than 30 studies analyzed the possible diagnostic

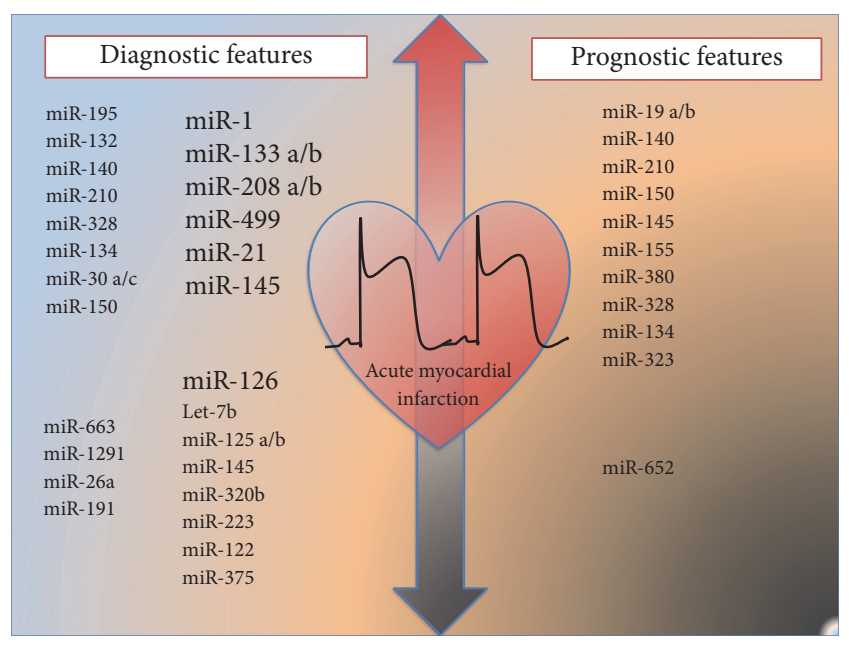

FIgURE 2: Circulating microRNAs associated with diagnostic and prognostic features in acute coronary syndrome (ACS). microRNAs in bigger font have been associated with ACS in more than one study. See Table 1 for further details.

microRNAs signature in AMI and other possible miRNA candidates have been proposed, but further validation studies are needed [26]. Years later, almost the same miRNAs are recognized as cardiac-enriched and proposed as possible biomarkers among several other miRNAs by 2 different authors. In 2014, a meta-analysis [27] of 19 studies evaluated the specificity and sensitivity of miR-1, miR-133a, miR-208b, and miR-499 in AMI. Cheng et al. concluded that miR-499 and miR-133a are possible biomarkers of AMI, showing a sensitivity of 0.88 (95\% CI: $0.86-0.90 ; p=0.0000)$; a specificity of 0.87 (95\% CI: $0.84-0.90 ; p=0.0000$ ) and a sensitivity of 0.89 (95\% CI: $0.83-0.94 ; p=0.0047)$; a specificity of 0.87 (95\% CI: $0.79-0.92 ; p=0.0262$ ), respectively. More recently, in a systematic review [28] the authors proposed that only cardiomyocyte-enriched miRNAS, miR-1, miR-133a/b, miR145, miR-208a/b, and miR-499(a) in plasma and/or serum are potential biomarkers for the diagnosis of coronary heart disease.

Devaux et al. [29] presented the largest multicenter study on miRNAs in 1155 unselected patients with acute chest pain. miR-208b provided the highest diagnostic accuracy in AMI but still this was lower than that of the fourth-generation or high-sensitivity cardiac troponin $\mathrm{T}(\mathrm{c} T \mathrm{Tn} T)$. None of the six miRNAs provided added diagnostic value when combined with cTnT.

The prognostic role of miRNAs is encouraging. Few studies [29-34] have evaluated the role of miRNAs as prognostic biomarkers with controversial results; see Table 1 for further details. Very recently Karakas et al. [34] found for the first time that peripheral-blood miRNAs (miR-132, miR140-3p, and miR-210) could predict CV mortality in a large cohort of ACS and stable CAD patients, while none of the cardiomyocyte-enriched miRNAs evaluated by Devaux et al. [29] predicted long-term mortality at 2-year follow-up, neither miR-208b nor miR-499 were significant predictors of mortality [33, 35]. Widera et al. [31, 34] found that miR-133a 


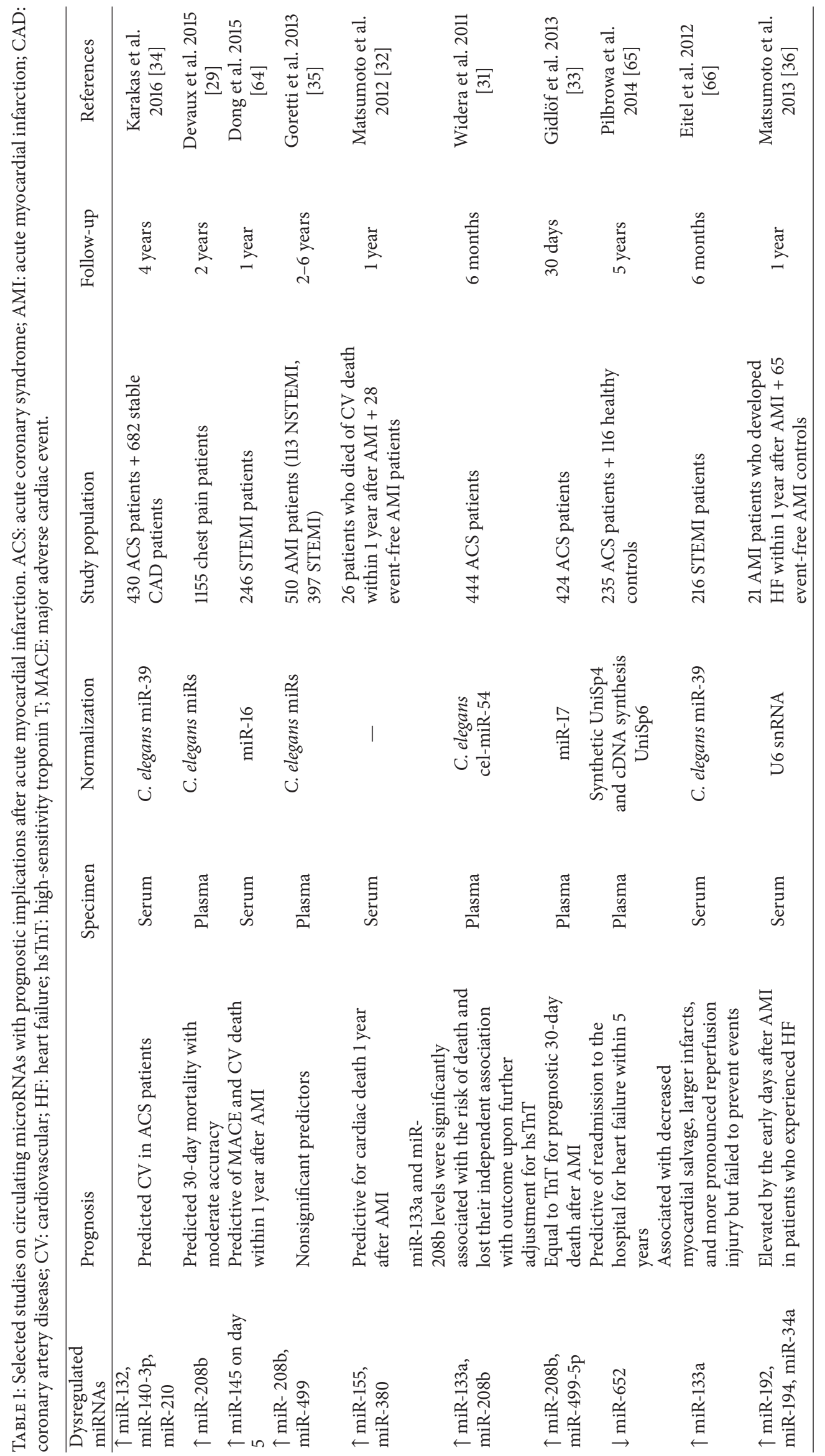


and miR- 208b levels were significantly associated with the risk of death in ACS patients, but in adjusted analysis their independent association with outcome was lost. Matsumoto et al. $[32,36]$ proposed 2 different sets of miRNAs with prognostic implications at 1-year follow-up post-AMI, but validation studies are needed for both.

3.2. Controversies in the Role of miRNAs as Biomarkers of $A M I$. The role of miRNAs has novel biomarkers in the early diagnosis of AMI is debated. The index test is high-sensitivity cardiac troponin, which is widely used in clinical practice and shows high accuracy in AMI diagnosis; therefore, it is very difficult for new biomarkers to demonstrate significant added value on top of cardiac troponins. Moreover, the 3rd universal definition of AMI relies on symptoms and detection of troponin-positive myocardial necrosis, even if the unspecific elevation of troponin levels can be present in case of nonischemic heart failure (HF), renal failure, myocarditis, arrhythmias, and pulmonary embolism due to myocardial injury [37]. Wang et al. [25] reported that, in AMI patients, miR-208 levels were not altered by chronic kidney disease or trauma, as it happens for troponins. Actually miRNAs can reduce this gap and provide additional accuracy in the diagnosis of AMI, as some miRNAs became detectable when initial troponin was still negative or within $3 \mathrm{~h}$ of symptom onset [38]. De iure miRNAs become detectable earlier than highsensitivity troponin, theoretically allowing a faster rule-in/ rule-out of chest pain patients; one of the major limits of $c \mathrm{TnT}$ is that multiple dosage at different time is needed and patients are ordered to stay in the emergency room for 3-6 h after arrival. De facto measurement of circulating miRNAs requires qRT-PCR, which is a time-consuming technique, in comparison with detection of hs-cTnT (approximately 30 $\mathrm{min}$ ) and the 2015 ESC guidelines recommend the use of a rapid rule-out protocol $(0 \mathrm{~h}$ and $1 \mathrm{~h}$ or 0 and $3 \mathrm{~h})$ when hscTnT is available [39]. The use of qRT-PCR is currently the limiting factor in terms of rapid detection of circulating miRNAs. In the future, the availability of newer, faster, and cost-effective techniques may overcome this limit.

3.3. MicroRNAs in Coronary Artery Disease. The ability to distinguish stable from unstable angina pectoris patients would be a great advance in CAD management, but this promise is far from being fulfilled, as concluded by D'Alessandra et al. [40]. Several miRNAs, as cardiomyocyteenriched (miR-133, miR-208a) [41], endothelial cell-enriched (miR-126, miR-17-92a cluster), vascular smooth cell (miR143/145) and inflammatory cell-enriched (miR-155), and platelet-enriched (miR-199a) miRNAs, were associated with CAD, while lipometabolism-related miR-122 and miR-370 increased as the severity of CAD quantified by the Gensini score increased [42]. Previously, miR-126 has been proposed as a prognostic marker of incident myocardial infarction in the general population [43], result partially confirmed by Jansen et al. [44] who reported that only microvesiclesassociated miR-126 and miR-199a predict the occurrence of $\mathrm{CV}$ events in patients with stable CAD. A more comprehensive review has been recently published [45].

\section{Technical Aspects of miRNAs Quantification}

The sensible differences and heterogeneous results reported in ACS and CAD studies can be partly explained by some technical aspects and drugs interaction. Quantification of miRNAs transcripts by qRT-PCR implicates data normalization with endogenous and exogenous reference genes for data correction. Data from qRT-PCR can be analyzed using absolute or relative quantification. Absolute quantification defines expression levels in absolute numbers of copies by relaying the PCR signal to a standard curve. Relative quantification determines fold changes in expression between two samples, normalizing the gene of interest for a housekeeping gene in the same sample to obtain a fold change. One of the most frequently used normalizers is the small noncoding RNA RNU6, which is not a miRNA and could not perfectly reflect the miRNAs biochemical characteristics. miR-16 is another frequently used normalizer because it is highly expressed and relatively invariant. The choice of the reference gene can be challenging as an optimal normalization strategy is missing. Consequently, the choice of which miRNAs should be used as internal controls for circulating miRNAs assessment could lead to ambiguous data interpretation, misleading conclusions, and erroneous biological predicted effect, impairing comparison between studies and meta-analysis of data. The use of more than 1 reference gene increases the accuracy of quantification; for example, the combined use of miR-16 and other miRNAs could reduce the potential bias compared to the use of a single reference gene [46]. Some authors stated that, in the lack of a shared housekeeping miRNA, miRNAs expressions do not require an internal control and could be normalized to serum volume [47]. However, this strategy has been demonstrated to increase the risk of bias and should be avoided. In addition, while searching for the ideal normalization gene candidate, it is pivotal to apply standardization across laboratories for sample preservation, storage, and stability.

Another potentially confounding factor is drug administration. Statins [42], anticoagulation [48], and antiplatelet drugs [49] can affect quantification of miRNAs in blood samples and must be taken into account when assessing circulating miRNAs [50]. To overcome the potential confounding effect of heparin, Kaudewitz et al. suggested normalizing with exogenous C. elegans spike-in control [51]. Other options to treat plasma from patients subjected to heparin treatment include digestion with heparinase on purified RNA rather than plasma, optimization of the starting plasma volume, and enrichment of miRNA on silica [52, 53]. To successfully translate miRNA signature in clinical practice it is mandatory to develop and apply a standardization of the operative procedures related to circulating miRNAs analysis. Standardization needs to be applied at several stages, from blood withdrawal to plasma/serum centrifugation, to sample collection and banking, and to RNA extraction and microRNAs quantification, in order to dramatically reduce interlaboratory differences that could generate huge incoherencies in miRNAs analyses. Consequently, bias in the selection, extraction, and quantification of miRNAs generating unexplained 
TABLE 2: Items reviewed on observational studies assessing the value of microRNAs as potential biomarkers for coronary artery disease and acute coronary syndrome. Table adapted by authors from [55].

\begin{tabular}{|c|c|c|}
\hline Item & Issue & Question \\
\hline (1) & Model assumption and goodness-of-fit & How far away from the data is the selected model? \\
\hline (2) & Interaction analysis & Is there any potential variable that can modify the estimated effect? \\
\hline (3) & Sensitivity analysis & Are the findings sufficiently robust, considering the process used to obtain them? \\
\hline (4) & Crude and adjusted effect estimate & $\begin{array}{l}\text { How much does the studied effect change when other variables are taken into } \\
\text { account? }\end{array}$ \\
\hline (5) & More than one adjusted model specified & $\begin{array}{l}\text { Does the estimated effect differ between the different adjusted models, settings, } \\
\text { specifications, and so forth? }\end{array}$ \\
\hline
\end{tabular}

contrasting results is widespread in most studies and represent a major limitation to perform a meta-analysis.

\section{Quality Reporting in Circulating MicroRNAs Observational Studies in Coronary Heart Disease}

Several authors reported that the biggest limitation for use of miRNAs as biomarkers is the small sample size of published studies $[48,54]$. Not only small sample size, but also the quality of reporting of observational studies is a major issue, due to the lack of randomized double blind trials. Controlling for already mentioned confounders is a crucial step in microRNAs observational studies, to avoid misleading conclusion. To overcome this problem, the use of multivariable models as statistical adjustment techniques is widely encouraged and the validation of assumption of the multivariable regression models should be clearly stated in the methodology. To our knowledge, a quality report on statistical methodology in circulating miRNAs studies has not yet been performed. We reviewed the quality of statistical reporting of 56 studies (see Supplementary Figure S1 and Table S1 for included studies in Supplementary Material available online at http://dx.doi.org/10.1155/2016/2150763) on circulating miRNAs in coronary heart disease (ACS, AMI, and CAD). A list of the Real et al. items reviewed in full-text studies is presented in Table 2, based on Real et al. methods [55]. See Supplementary Materials for complete methods and statistical analysis. Results are resumed in Table 3. The large majority of studies are in fact cohort studies of small sample size (median size 115 patients). Of note, significant differences exist between small ( $<100$ patients) and large sample size (>500 patients) studies in terms of quality reporting of multivariable regression models. A multiple regression was run to predict a quality score $>2$ from adopted model, journal impact factor, citation/year, and sample size. Among these variables, only sample size statistically significantly predicted quality score, $F(5,50)=22.201, p<0.0001, R^{2}=.689$. The totality of larger sample size studies scored at least 3 over 5 checked items, demonstrating a solid methodology and control for confounders. In fact, some miRNAs lost statistical significance when adjusted for confounders [29, 34, 43]. It is highly possible that among the authors of large sample size study a methodologist is included. Small sample size studies without adjustment for confounders of the results contribute to increase heterogeneity and introduce possible bias in the literature. No significant differences exist in terms of article citations per year; highly cited articles can have a robust or a weak methodology. Obviously the first reports were small sample size studies but great breakthroughs in microRNAs biology and function were therefore highly cited. Studies with weak methodology can present contrasting results and then be cited in contrast to more robust studies, creating confusion. Nevertheless, the journal impact factor has definitely a role in assessing the methodological quality of the study and even if it does not reach the full significance in our results, the trend is in favor of a positive correlation between impact factor and high methodological score.

\section{MicroRNA-Based Therapeutics}

Up-to-date microRNAs-based therapies are in their infancy, thus experimental and animal studies are in favor of a potential role in the treatment of CV diseases. In nonhuman primates, inhibition of miR-33a and miR-33b by an anti-miRNA oligonucleotide increased hepatic expression of ABCA1, a key regulator of high density lipoprotein (HDL) biogenesis, and induced a sustained increase in plasma HDL levels over 12 weeks, with reduction of very low density lipoprotein (VLDL) levels [56]. Another study assessed the role of locked nucleic acid-modified antisense miR-92a (LNA-92a) in a model of ischemia/reperfusion injury in pigs and revealed cellprotective, proangiogenic, and anti-inflammatory effects of LNA-92a with reduction of infarct size and improved recovery of cardiac function [57]. Unfortunately these promising results have not yet progressed to human trials. After the seminal studies on miR-21 by Thum [58], a key target in CV diseases would be reduction/inhibition of myocardial fibrosis associated with postischemic cardiopathy, drug-induced or primitive cardiomyopathies [59], but the question is far from being resolved yet $[60,61]$. In other fields of medicine miRNA-based therapies are a step forward. In patients with chronic hepatitis $\mathrm{C}$, subcutaneous administration of an antisense oligonucleotide for miR-122 led to successful results with negligible side effects in phases 1 and $2 \mathrm{a}$ trial [62] and at long-term follow-up [63]. 


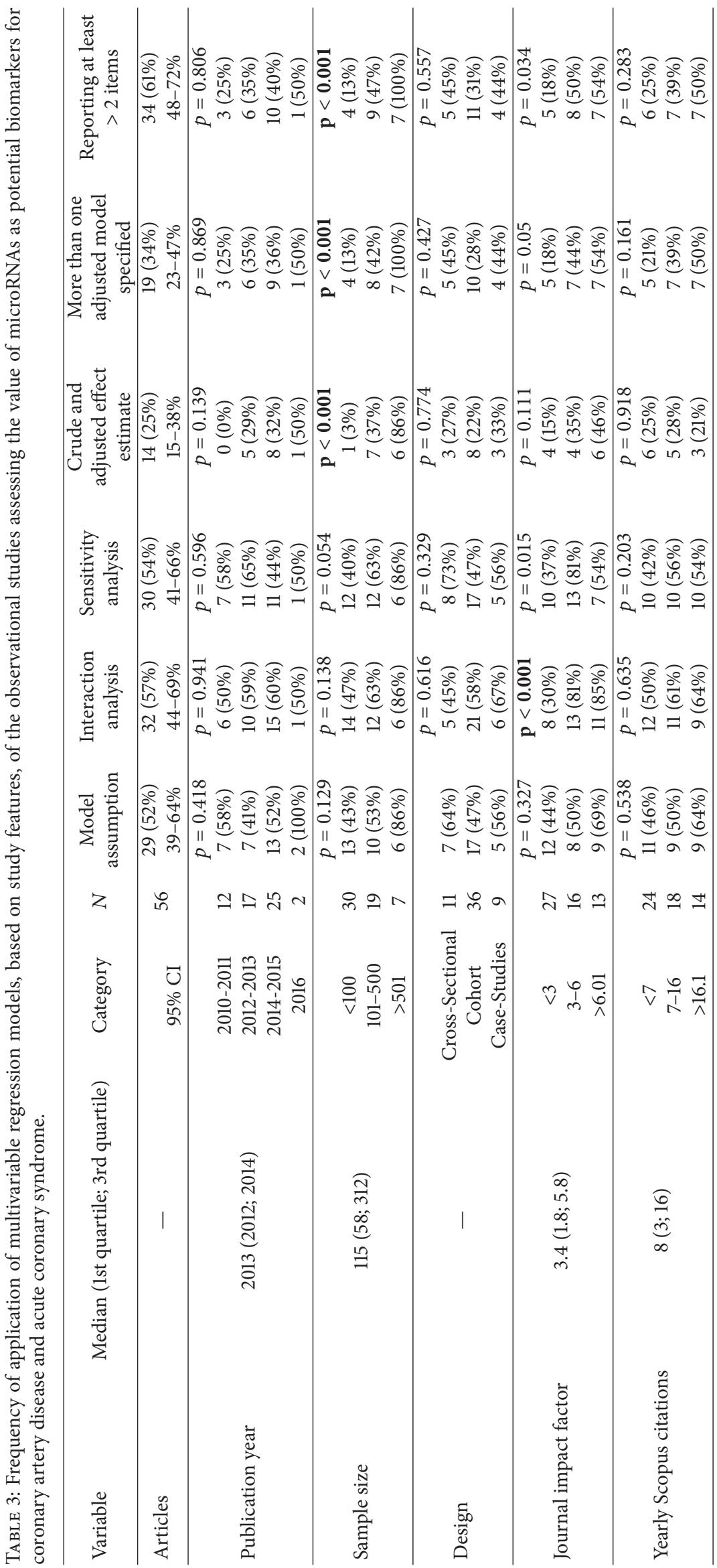




\section{Conclusion}

Translational research represents a stem of scientific research that helps to make findings from basic science useful for practical applications that enhance human health and wellbeing. Deeply established on multidisciplinary collaboration, translational research has the enormous potential to move applied science forward. Accordingly, in the cardiovascular system, miRNAs fine-tune complex molecular signaling networks by acting on key target proteins involved in a variety of pathways and cellular processes. Therefore, in the past decade, several studies emphasized the importance of miRNAs as diagnostic and prognostic role in cardiovascular disease and the road travelled so far seems promising for a specific role in coronary heart disease. At present, circulating miRNAs have not entered yet the clinical arena, due to contrasting results, possible confounding factors, presence of small or moderately sized studies of different methodology, sometimes challenging each other, technological requirements, and unstandardized normalization. This complex scenario, in which bordering results contradict themselves, may push researchers, clinicians, and also patients in different directions providing dissimilar effect estimates with mixed results and with benefits ranging from absent to transient and, at most, marginal. In the future, the role of long noncoding RNAs may add novel insight into the posttranscriptional regulation changing the way with which investigators identify novel signal transduction pathways and functional crosstalks developing new therapeutic strategies and micro-RNA based therapies might make the way for human trials with important therapeutic implications. Clinicians must be aware of the pros and cons of microRNAs advent and read critically the fore coming literature.

\section{Abbreviations}

ACS: $\quad$ Acute coronary syndrome

AMI: Acute myocardial infarction

CAD: $\quad$ Coronary artery disease

CV: $\quad$ Cardiovascular

DGCR8: Di George syndrome critical region 8

HDL: High density lipoprotein

hs-cTNT: High-sensitivity cardiac troponin T

LNA: $\quad$ Locked nucleic acid

miRNA: MicroRNA

mRNA: Messenger RNA

qRT-PCR: Quantitative real-time polymerase chain reaction

TRBP: TAR RNA-binding protein

VLDL: Very low density lipoprotein.

\section{Competing Interests}

The authors declare that they have no competing interests.

\section{Acknowledgments}

The authors thank Dr. Daniele Cuminale for his invaluable support and help with the graphic design. This work was supported by Sapienza University of Rome (Project C26A14258W) (awarded to Elena Cavarretta).

\section{References}

[1] D. Mozaffarian, E. J. Benjamin, A. S. Go et al., "American heart association statistics committee; Stroke statistics subcommittee. Heart disease and stroke statistics-2016 update: a report from the American Heart Association," Circulation, vol. 133, no. 4, pp. e38-e60, 2016.

[2] J. D. Berry, A. Dyer, X. Cai et al., "Lifetime risks of cardiovascular disease," The New England Journal of Medicine, vol. 366, no. 4, pp. 321-329, 2012.

[3] S. Zdravkovic, A. Wienke, N. L. Pedersen, M. E. Marenberg, A. I. Yashin, and U. De Faire, "Heritability of death from coronary heart disease: a 36-year follow-up of 20966 Swedish twins," Journal of Internal Medicine, vol. 252, no. 3, pp. 247-254, 2002.

[4] N. V. Rivera, R. Carreras-Torres, R. Roncarati et al., "Assessment of the 9p21.3 locus in severity of coronary artery disease in the presence and absence of type 2 diabetes," BMC Medical Genetics, vol. 14, no. 1, article 11, 2013.

[5] R. McPherson and A. Tybjaerg-Hansen, "Genetics of coronary artery disease," Circulation Research, vol. 118, no. 4, pp. 564-578, 2016.

[6] A. Busch, S. M. Eken, and L. Maegdefessel, "Prospective and therapeutic screening value of non-coding RNA as biomarkers in cardiovascular disease," Annals of Translational Medicine, vol. 4, no. 12, pp. 236-236, 2016.

[7] R. C. Lee, R. L. Feinbaum, and V. Ambros, "The C. elegans heterochronic gene lin-4 encodes small RNAs with antisense complementarity to lin-14," Cell, vol. 75, no. 5, pp. 843-854, 1993.

[8] D. P. Bartel, "MicroRNAs: genomics, biogenesis, mechanism, and function," Cell, vol. 116, no. 2, pp. 281-297, 2004.

[9] B. J. Reinhart, F. J. Slack, M. Basson et al., "The 21-nucleotide let-7 RNA regulates developmental timing in Caenorhabditis elegans," Nature, vol. 403, no. 6772, pp. 901-906, 2000.

[10] A. Kozomara and S. Griffiths-Jones, "MiRBase: annotating high confidence microRNAs using deep sequencing data," Nucleic Acids Research, vol. 42, no. 1, pp. D68-D73, 2014.

[11] G. Condorelli, M. V. G. Latronico, and E. Cavarretta, "MicroRNAs in cardiovascular diseases: current knowledge and the road ahead," Journal of the American College of Cardiology, vol. 63, no. 21, pp. 2177-2187, 2014.

[12] H. A. Meijer, E. M. Smith, and M. Bushell, "Regulation of miRNA strand selection: follow the leader?" Biochemical Society Transactions, vol. 42, no. 4, pp. 1135-1140, 2014.

[13] M. Ha and V. N. Kim, "Regulation of microRNA biogenesis," Nature Reviews Molecular Cell Biology, vol. 15, no. 8, pp. 509524, 2014.

[14] R. A. Boon and K. C. Vickers, "Intercellular transport of MicroRNAs," Arteriosclerosis, Thrombosis, and Vascular Biology, vol. 33, no. 2, pp. 186-192, 2013.

[15] H. Zhu and G. C. Fan, "Extracellular/circulating microRNAs and their potential role in cardiovascular disease," American Journal of Cardiovascular Disease, vol. 1, no. 2, pp. 138-149, 2011.

[16] P. S. Mitchell, R. K. Parkin, E. M. Kroh et al., "Circulating microRNAs as stable blood-based markers for cancer detection," Proceedings of the National Academy of Sciences of the United States of America, vol. 105, no. 30, pp. 10513-10518, 2008.

[17] N. Kosaka, H. Izumi, K. Sekine, and T. Ochiya, "MicroRNA as a new immune-regulatory agent in breast milk," Silence, vol. 1, no. 1, article 7, 2010. 
[18] J. A. Weber, D. H. Baxter, S. Zhang et al., "The microRNA spectrum in 12 body fluids," Clinical Chemistry, vol. 56, no. 11, pp. 1733-1741, 2010.

[19] A. Turchinovich, L. Weiz, A. Langheinz, and B. Burwinkel, "Characterization of extracellular circulating microRNA," Nucleic Acids Research, vol. 39, no. 16, pp. 7223-7233, 2011.

[20] N. B. Y. Tsui, E. K. O. Ng, and Y. M. D. Lo, "Stability of endogenous and added RNA in blood specimens, serum, and plasma," Clinical Chemistry, vol. 48, no. 10, pp. 1647-1653, 2002.

[21] J. Ai, R. Zhang, Y. Li et al., "Circulating microRNA-1 as a potential novel biomarker for acute myocardial infarction," Biochemical and Biophysical Research Communications, vol. 391, no. 1, pp. 73-77, 2010.

[22] Y. D’Alessandra, P. Devanna, F. Limana et al., "Circulating microRNAs are new and sensitive biomarkers of myocardial infarction," European Heart Journal, vol. 31, no. 22, pp. 27652773, 2010.

[23] M. F. Corsten, R. Dennert, S. Jochems et al., "Circulating MicroRNA-208b and MicroRNA-499 reflect myocardial damage in cardiovascular disease," Circulation: Cardiovascular Genetics, vol. 3, no. 6, pp. 499-506, 2010.

[24] T. Adachi, M. Nakanishi, Y. Otsuka et al., "Plasma microRNA 499 as a biomarker of acute myocardial infarction," Clinical Chemistry, vol. 56, no. 7, pp. 1183-1185, 2010.

[25] G.-K. Wang, J.-Q. Zhu, J.-T. Zhang et al., "Circulating microRNA: a novel potential biomarker for early diagnosis of acute myocardial infarction in humans," European Heart Journal, vol. 31, no. 6, pp. 659-666, 2010.

[26] S. P. R. Romaine, M. Tomaszewski, G. Condorelli, and N. J. Samani, "MicroRNAs in cardiovascular disease: an introduction for clinicians," Heart, vol. 101, no. 12, pp. 921-928, 2015.

[27] C. Cheng, Q. Wang, W. You, M. Chen, and J. Xia, "MiRNAs as biomarkers of myocardial infarction: a meta-analysis," PLoS ONE, vol. 9, no. 2, Article ID e88566, 2014.

[28] R. Navickas, D. Gal, A. Laucevičius, A. Taparauskaitè, M. Zdanyte, and P. Holvoet, "Identifying circulating microRNAs as biomarkers of cardiovascular disease: a systematic review," Cardiovascular Research, vol. 111, no. 4, pp. 322-337, 2016.

[29] Y. Devaux, M. Mueller, P. Haaf et al., "Diagnostic and prognostic value of circulating microRNAs in patients with acute chest pain," Journal of Internal Medicine, vol. 277, no. 2, pp. 260-271, 2015.

[30] F. He, P. Lv, X. Zhao et al., "Predictive value of circulating miR328 and miR-134 for acute myocardial infarction," Molecular and Cellular Biochemistry, vol. 394, no. 1-2, pp. 137-144, 2014.

[31] C. Widera, S. K. Gupta, J. M. Lorenzen et al., "Diagnostic and prognostic impact of six circulating microRNAs in acute coronary syndrome," Journal of Molecular and Cellular Cardiology, vol. 51, no. 5, pp. 872-875, 2011.

[32] S. Matsumoto, Y. Sakata, D. Nakatani et al., "A subset of circulating microRNAs are predictive for cardiac death after discharge for acute myocardial infarction," Biochemical and Biophysical Research Communications, vol. 427, no. 2, pp. 280284, 2012.

[33] O. Gidlöf, J. G. Smith, K. Miyazu et al., "Circulating cardioenriched microRNAs are associated with long-term prognosis following myocardial infarction," BMC Cardiovascular Disorders, vol. 13, article 12, 2013.

[34] M. Karakas, C. Schulte, S. Appelbaum et al., "Circulating microRNAs strongly predict cardiovascular death in patients with coronary artery disease-results from the large AtheroGene study," European Heart Journal, 2016.
[35] E. Goretti, M. Vausort, D. R. Wagner, and Y. Devaux, "Association between circulating microRNAs, cardiovascular risk factors and outcome in patients with acute myocardial infarction," International Journal of Cardiology, vol. 168, no. 4, pp. 45484550, 2013.

[36] S. Matsumoto, Y. Sakata, S. Suna et al., "Circulating p53responsive MicroRNAs are predictive indicators of heart failure after acute myocardial infarction," Circulation Research, vol. 113, no. 3, pp. 322-326, 2013.

[37] K. Thygesen, J. S. Alpert, A. S. Jaffe, M. L. Simoons, B. R. Chaitman, and H. D. White, "Writing Group on behalf of the Joint ESC/ACCF/AHA/WHF Task Force for the Universal Definition of Myocardial Infarction. Third universal definition of myocardial infarction," Circulation, vol. 126, pp. 2020-2035, 2012.

[38] M. I. F. J. Oerlemans, A. Mosterd, M. S. Dekker et al., "Early assessment of acute coronary syndromes in the emergency department: the potential diagnostic value of circulating microRNAs," EMBO Molecular Medicine, vol. 4, no. 11, pp. 11761185, 2012.

[39] M. Roffi, C. Patrono, J. P. Collet et al., "Management of Acute Coronary Syndromes in Patients Presenting without Persistent ST-Segment Elevation of the European Society of Cardiology. 2015 ESC Guidelines for the management of acute coronary syndromes in patients presenting without persistent ST-segment elevation: Task Force for the Management of Acute Coronary Syndromes in Patients Presenting without Persistent ST-Segment Elevation of the European Society of Cardiology (ESC)," European Heart Journal, vol. 37, no. 3, pp. 267-315, 2016.

[40] Y. D’Alessandra, M. C. Carena, L. Spazzafumo et al., "Diagnostic potential of plasmatic microRNA signatures in stable and unstable angina," PLoS ONE, vol. 8, no. 11, Article ID e80345, 2013.

[41] S. Fichtlscherer, S. De Rosa, H. Fox et al., "Circulating microRNAs in patients with coronary artery disease," Circulation Research, vol. 107, no. 5, pp. 677-684, 2010.

[42] W. Gao, H.-W. He, Z.-M. Wang et al., "Plasma levels of lipometabolism-related miR-122 and miR-370 are increased in patients with hyperlipidemia and associated with coronary artery disease," Lipids in Health and Disease, vol. 11, article 55, 2012.

[43] A. Zampetaki, P. Willeit, L. Tilling et al., "Prospective study on circulating microRNAs and risk of myocardial infarction," Journal of the American College of Cardiology, vol. 60, no. 4, pp. 290-299, 2012.

[44] F. Jansen, X. Yang, S. Proebsting et al., "MicroRNA expression in circulating microvesicles predicts cardiovascular events in patients with coronary artery disease," Journal of the American Heart Association, vol. 3, no. 6, article e001249, 2014.

[45] E. K. Economou, E. Oikonomou, G. Siasos et al., "The role of microRNAs in coronary artery disease: from pathophysiology to diagnosis and treatment," Atherosclerosis, vol. 241, no. 2, pp. 624-633, 2015.

[46] H. Schwarzenbach, A. M. da Silva, G. Calin, and K. Pantel, "Data normalization strategies for microRNA quantification," Clinical Chemistry, vol. 61, no. 11, pp. 1333-1342, 2015.

[47] J. Wang, Y. Pei, Y. Zhong, S. Jiang, J. Shao, and J. Gong, "Altered serum microRNAs as novel diagnostic biomarkers for atypical coronary artery disease," PLoS ONE, vol. 9, no. 9, Article ID e107012, 2014.

[48] J.-N. Boeckel, C. E. Thomé, D. Leistner, A. M. Zeiher, S. Fichtlscherer, and S. Dimmeler, "Heparin selectively affects the 
quantification of micrornas in human blood samples," Clinical Chemistry, vol. 59, no. 7, pp. 1125-1127, 2013.

[49] H. C. de Boer, C. van Solingen, J. Prins et al., "Aspirin treatment hampers the use of plasma microRNA-126 as a biomarker for the progression of vascular disease," European Heart Journal, vol. 34, no. 44, pp. 3451-3457, 2013.

[50] E. Cavarretta, G. A. Chiariello, and G. Condorelli, "Platelets, endothelium, and circulating microRNA-126 as a prognostic biomarker in cardiovascular diseases: per aspirin ad astra," European Heart Journal, vol. 34, no. 44, pp. 3400-3402, 2013.

[51] D. Kaudewitz, A. Zampetaki, and M. Mayr, "MicroRNA biomarkers for coronary artery disease?" Current Atherosclerosis Reports, vol. 17, no. 12, article 70, 2015.

[52] D.-J. Kim, S. Linnstaedt, J. Palma et al., "Plasma components affect accuracy of circulating cancer-related microRNA quantitation," Journal of Molecular Diagnostics, vol. 14, no. 1, pp. 71-80, 2012.

[53] P. Tiberio, M. Callari, V. Angeloni, M. G. Daidone, and V. Appierto, "Challenges in using circulating miRNAs as cancer biomarkers," BioMed Research International, vol. 2015, Article ID 731479, 10 pages, 2015.

[54] R. Kumarswamy and T. Thum, "Non-coding RNAs in cardiac remodeling and heart failure," Circulation Research, vol. 113, no. 6, pp. 676-689, 2013.

[55] J. Real, C. Forné, A. Roso-Llorach, and J. M. Martínez-Sánchez, "Quality reporting of multivariable regression models in observational studies," Medicine, vol. 95, no. 20, article e3653, 2016.

[56] K. J. Rayner, C. C. Esau, F. N. Hussain et al., "Inhibition of miR$33 \mathrm{a} / \mathrm{b}$ in non-human primates raises plasma HDL and lowers VLDL triglycerides," Nature, vol. 478, no. 7369, pp. 404-407, 2011.

[57] R. Hinkel, D. Penzkofer, S. Zühlke et al., "Inhibition of microRNA-92a protects against ischemia/reperfusion injury in a large-animal model," Circulation, vol. 128, no. 10, pp. 10661075, 2013.

[58] T. Thum, C. Gross, J. Fiedler et al., "MicroRNA-21 contributes to myocardial disease by stimulating MAP kinase signalling in fibroblasts," Nature, vol. 456, no. 7224, pp. 980-984, 2008.

[59] M. Lotrionte, E. Cavarretta, A. Abbate et al., “Temporal changes in standard and tissue doppler imaging echocardiographic parameters after anthracycline chemotherapy in women with breast cancer," American Journal of Cardiology, vol. 112, no. 7, pp. 1005-1012, 2013.

[60] E. Cavarretta and G. Condorelli, "miR-21 and cardiac fibrosis: another brick in the wall?" European Heart Journal, vol. 36, no. 32, pp. 2139-2141, 2015.

[61] E. Cavarretta, M. V. G. Latronico, and G. Condorelli, "Endothelial-to-mesenchymal transition and microRNA-21: the game is on again," Arteriosclerosis, Thrombosis, and Vascular Biology, vol. 32, no. 2, pp. 165-166, 2012.

[62] H. L. A. Janssen, H. W. Reesink, E. J. Lawitz et al., "Treatment of HCV infection by targeting microRNA," New England Journal of Medicine, vol. 368, no. 18, pp. 1685-1694, 2013.

[63] M. H. van der Ree, A. J. van der Meer, J. de Bruijne et al., "Long-term safety and efficacy of microRNA-targeted therapy in chronic hepatitis C patients," Antiviral Research, vol. 111, pp. 53-59, 2014.

[64] Y.-M. Dong, X.-X. Liu, G.-Q. Wei, Y.-N. Da, L. Cha, and C.-S. $\mathrm{Ma}$, "Prediction of long-term outcome after acute myocardial infarction using circulating miR-145," Scandinavian Journal of Clinical and Laboratory Investigation, vol. 75, no. 1, pp. 85-91, 2015.
[65] A. P. Pilbrowa, L. Cordeddu, V. A. Cameron et al., "Circulating miR-323-3p and miR-652: candidate markers for the presence and progression of acute coronary syndromes," International Journal of Cardiology, vol. 176, no. 2, pp. 375-385, 2014.

[66] I. Eitel, V. Adams, P. Dieterich et al., "Relation of circulating MicroRNA-133a concentrations with myocardial damage and clinical prognosis in ST-elevation myocardial infarction," American Heart Journal, vol. 164, no. 5, pp. 706-714, 2012. 


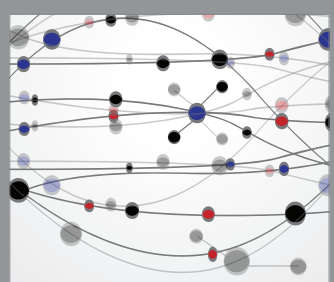

The Scientific World Journal
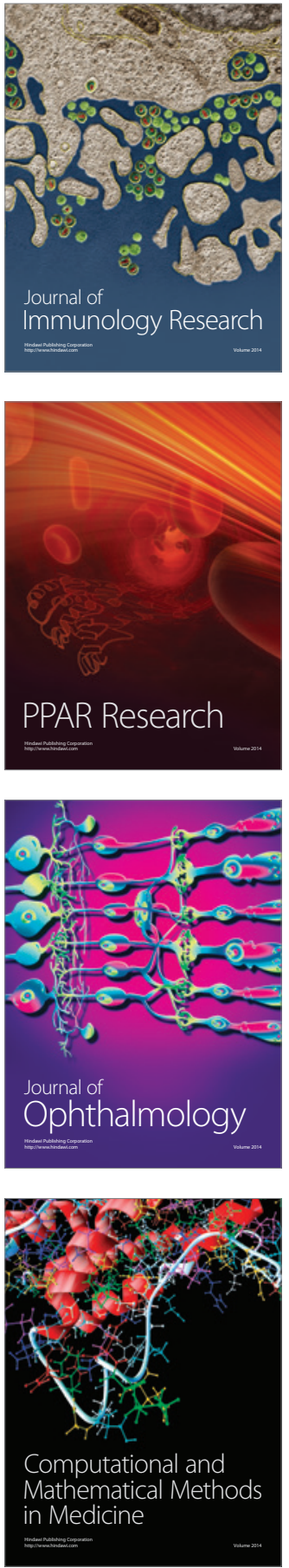

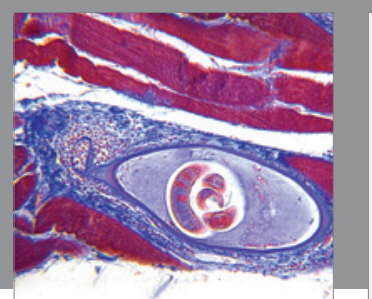

Gastroenterology Research and Practice

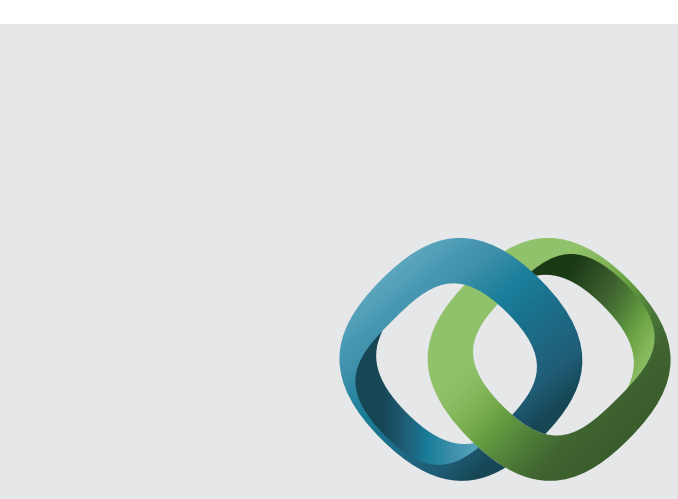

\section{Hindawi}

Submit your manuscripts at

http://www.hindawi.com
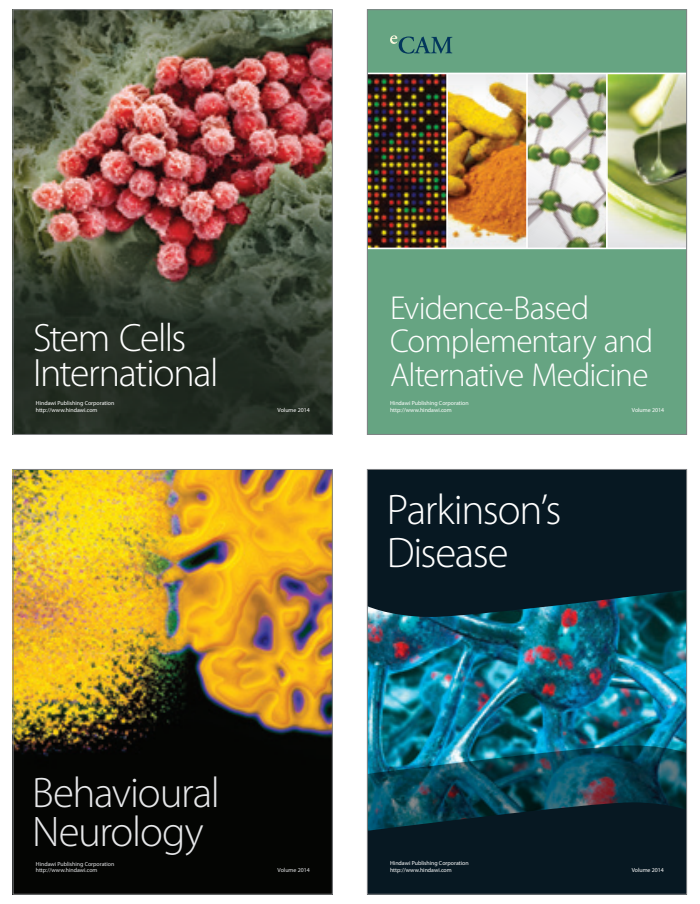
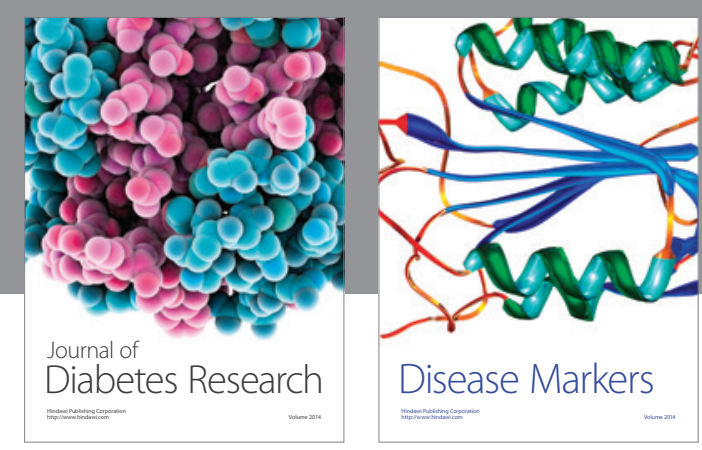

Disease Markers
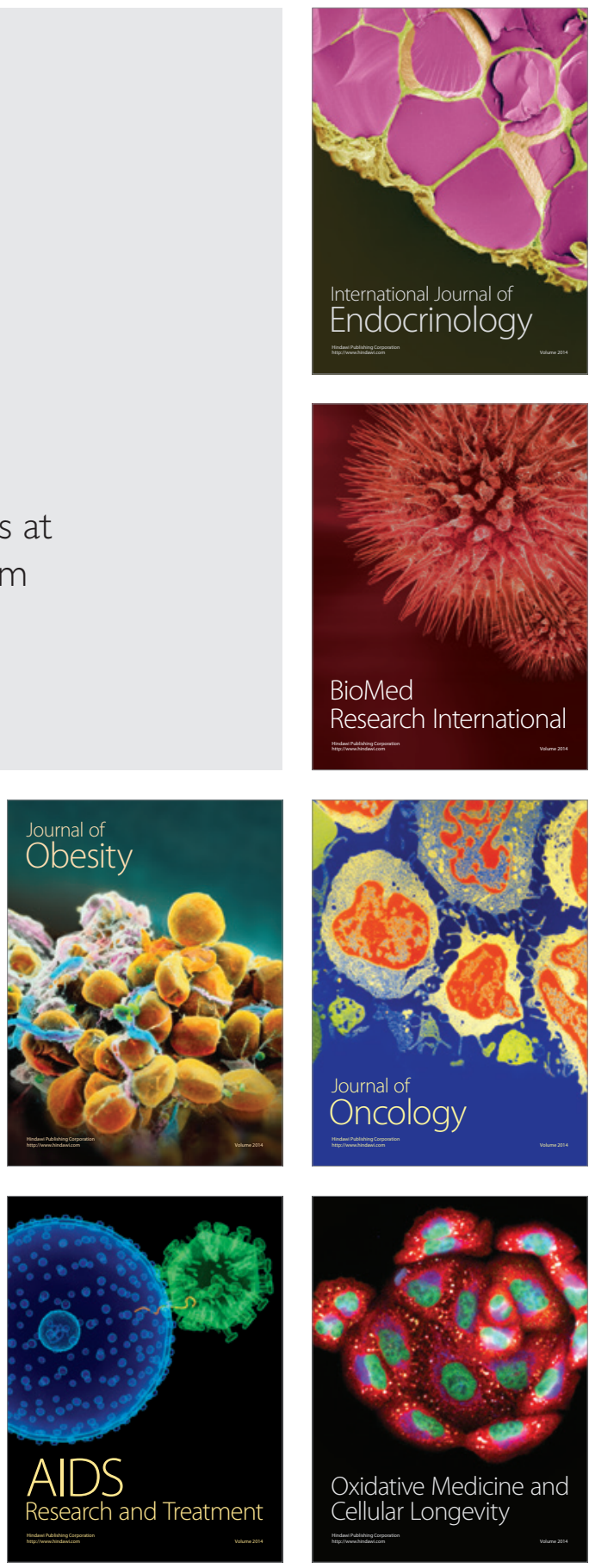